\section{Gute Laborpraxis}

U. Zimmermann ${ }^{1}$ und A. Steinhorst ${ }^{2}$

${ }^{1}$ Gesundheit/Forensik, Deutsche Akkreditierungsstelle

GmbH (DAkkS), Frankfurt, Deutschland

${ }^{2}$ EA Secretariat, Paris, Frankreich

\section{$\operatorname{Synonym(e)~GLP~}$}

\section{Englischer Begriff good laboratory practice (GLP)}

Definition Die Gute Laborpraxis (GLP) ist ein Qualitätssicherungssystem, das sich mit dem organisatorischen Ablauf und den Rahmenbedingungen befasst, unter denen nicht klinische gesundheits- und umweltrelevante Sicherheitsprüfungen geplant, durchgeführt und überwacht werden sowie mit der Aufzeichnung, Archivierung und Berichterstattung der Prüfungen.

Beschreibung Behörden und Industrie sind um die Qualität von nicht klinischen gesundheits- und umweltrelevanten Sicherheitsprüfungen, als Basis für eine Risikobewertung/Gefahrenabschätzung bemüht. Infolgedessen haben die OECDMitgliedstaaten Kriterien für die Durchführung dieser Prüfungen aufgestellt. Um unterschiedliche Verfahrensweisen bei der Umsetzung zu vermeiden, die den internationalen Handel mit Chemikalien behindern könnten, haben die OECD-Mitgliedstaaten im Jahr 1978 die „Grundsätze der Guten Laborpraxis“ entwickelt, basierend auf den GLP-Regularien für nicht klinische Prüfungen der US Food and Drug Administration (FDA). Diese GLP-Grundsätze wurden vom OECD-Rat im Jahr 1981 angenommen. In Deutschland sind die Grundsätze der Guten Laborpraxis Bestandteil des Chemikaliengesetzes (Anhang $1 \mathrm{zu} \S 19 \mathrm{a}$ Abs. 1).
Zweck der Grundsätze der Guten Laborpraxis ist es, die Qualität von Prüfdaten zu fördern. Die vergleichbare Qualität von Prüfdaten bildet die Grundlage für die gegenseitige Anerkennung der Daten unter den Ländern. Wenn einzelne Länder den in anderen Ländern gewonnenen Prüfdaten vertrauen können, lassen sich Doppelprüfungen vermeiden und dadurch Zeit und Ressourcen einsparen. Die Anwendung der Grundsätze soll technische Handelshemmnisse vermeiden helfen und den Schutz der menschlichen Gesundheit und der Umwelt weiter verbessern. Die Grundsätze der Guten Laborpraxis finden Anwendung auf die nicht klinischen Sicherheitsprüfungen von Prüfgegenständen, die in Arzneimitteln, Pflanzenschutzmitteln und Bioziden, kosmetischen Mitteln, Tierarzneimitteln sowie in Lebensmittelzusatzstoffen, Futtermittelzusatzstoffen und Industriechemikalien enthalten sind. Häufig sind diese Prüfgegenstände chemisch synthetisierte Produkte; sie können aber auch natürlichen bzw. biologischen Ursprungs sein; unter Umständen kann es sich um lebende Organismen handeln. Zweck der Prüfung dieser Prüfgegenstände ist es, Daten über deren Eigenschaften und/oder deren Unbedenklichkeit für die menschliche Gesundheit und/ oder die Umwelt zu gewinnen. $\mathrm{Zu}$ den nicht klinischen gesundheits- und umweltrelevanten Sicherheitsprüfungen, die durch die Grundsätze der Guten Laborpraxis abgedeckt werden, zählen sowohl Laborprüfungen als auch Prüfungen in Gewächshäusern oder im Freiland.

\section{Literatur}

Chemikaliengesetz in der Fassung der Bekanntmachung vom 28. August 2013 (BGBl. I S. 3498, 3991), das zuletzt durch Artikel 2 des Gesetzes vom 18. Juli 2017 (BGBl. I S. 2774) geändert worden ist

OECD-Dokumente zur Guten Laborpraxis; Nr. 1 OECD-Grundsätze der Guten Laborpraxis (Neufassung aus 1997) 